\title{
Dynamic hysteresis from zigzag domain walls: Discrete model and Monte Carlo simulations
}

\author{
Benedetta Cerruti and Stefano Zapperi \\ CNR-INFM SMC and Dipartimento di Fisica, Universitá "La Sapienza," P.le A. Moro 2, 00185 Roma, Italy
}

(Received 15 March 2006; revised manuscript received 16 October 2006; published 13 February 2007)

\begin{abstract}
We investigate dynamic hysteresis in ferromagnetic thin films with zigzag domain walls. We introduce a discrete model describing the motion of a wall in a disordered ferromagnet with in-plane magnetization, driven by an external magnetic field, considering the effects of dipolar interactions and anisotropy. We analyze the effects of external field frequency and temperature on the coercive field by Monte Carlo simulations, and find a good agreement with the experimental data reported in literature for Fe/GaAs films. This implies that dynamic hysteresis in this case can be explained by a single propagating domain wall model without invoking domain nucleation.
\end{abstract}

DOI: 10.1103/PhysRevB.75.064416

PACS number(s): 77.80.Dj, 75.60.Ch, 75.70.Ak

\section{INTRODUCTION}

Ferromagnetic materials are concrete examples of cooperatively interacting many-body systems. When a magnet is driven by a varying external magnetic field, the system cannot reach equilibrium instantaneously, due to the internal relaxation delay. If the external field oscillates, the magnetization will do so as well, lagging behind the field. This effect gives rise to a nonvanishing area of magnetization-field loop whose form will depend on the applied field frequency: a phenomenon known as dynamic hysteresis. ${ }^{1,2}$ Since the loop area represents the amount of externally supplied energy that is irreversibly transformed into heat during one magnetization cycle, dynamic hysteresis has important technological implications, e.g., for high frequency devices applications. Furthermore, from a purely theoretical point of view, the dynamics of disordered magnetic systems represents a central problem in nonequilibrium statistical mechanics. While in three dimensional systems dynamic hysteresis is well understood in terms of eddy currents dissipation, this effect is expected to become negligible by reducing the sample thickness. ${ }^{3}$ Thus recently there has been a renewed interest in understanding two dimensional systems, both experimentally ${ }^{2,4-17}$ and theoretically, ${ }^{1,18-20}$ motivated by the applications of thin ferromagnetic films in magnetic recording technology and spintronic devices.

Two classes of models are mostly used to investigate the magnetization reversal properties on a microscopic scale, spin models of the Ising type, ${ }^{1,22-24}$ or extended domain wall models. ${ }^{20,21,25}$ The theoretical tools used to interpret experimental data on dynamic hysteresis are often grown out of the first class of models which suggest a universal scaling law for the dependence of the hysteresis loop area $A$ on the external parameters, i.e., the temperature of the system $T$ and the amplitude $H_{0}$ and frequency $\omega$ of the applied magnetic field. In particular, it is expected from the models that $A$ $\propto H_{0}^{\alpha} \omega^{\beta} T^{-\gamma}$, where $\alpha, \beta$, and $\gamma$ are the scaling exponents. ${ }^{1}$ The experimental estimates of these exponents display, however, a huge variability ${ }^{4-12,17}$ and the validity of that universal scaling law is still under debate. ${ }^{16,26}$ Since various phenomena may in principle contribute to the hysteretic behavior, such as domain nucleation, domain wall propagation, or simply retardation of the magnetization due to fluc- tuations, it has been proposed that the lack of good scaling of the function $A(\omega)$ is due to a crossover between two distinct dynamical regimes, one dominated by domain wall propagation, and the other by nucleation of new domains. ${ }^{2,9}$

The second class of models used to investigate ferromagnetic systems considers the dynamics of individual domain walls as the relevant mechanism for hysteresis. In two dimensional systems, developing such a kind of model can be even more complicated than in the bulk three dimensional case, due the possibility for the magnetization to lay in or out the film plane, and the huge variety of domains and domain walls topologies (for an exhaustive overview of the existing configurations together with many experimental images, see Ref. 27). Dynamic hysteresis due to the motion of $180^{\circ}$ Bloch domain walls has been extensively investigated, ${ }^{16,20}$ but less is know about charged walls.

In this article we focus on two dimensional systems with zigzag domain walls, arising from the competition between dipolar forces and magnetocrystalline anisotropy in thin films with head-on magnetization between nearest-neighbor domains. ${ }^{28}$ These walls have been originally observed in thin film magnetic recording media, where head-on domains are induced by means of the application of a recording head field, and have been then reported in several magnetic materials such as iron, ${ }^{29} \mathrm{Co},{ }^{30} \mathrm{Gd}-\mathrm{Co},{ }^{28}$ epitaxial $\mathrm{Fe}$ films grown on $\operatorname{GaAs}(001),{ }^{9}$ finemets, and many others. In addition, zigzag walls have also been observed in ferroelectric materials, such as $\mathrm{Gd}_{2}\left(\mathrm{MoO}_{4}\right)_{3}$ crystals. ${ }^{31}$ Most calculations reported in literature for zigzag walls focus on the derivation of the equilibrium parameters (e.g., zigzag angle and amplitude or period) $)^{28,32}$ and do not consider their dynamics.

Here we introduce a discrete model for the motion of a single zigzag wall in a disordered ferromagnetic two dimensional sample with in-plane uniaxial magnetization, driven by an external (triangular) magnetic field. The model is based on the interplay between dipolar and anisotropy energy contributions, in the presence of structural disorder. Dynamic hysteresis is investigated by Monte Carlo simulations analyzing the behavior of the coercive field $H_{c}$ as a function of the external field frequency, temperature $T$, and sample thickness. We find a reasonable agreement with experimental data reported for $\mathrm{Fe} / \mathrm{GaAs}$ thin films. ${ }^{9}$ Our results indicate that the experiments can be interpreted by a domain wall propa- 


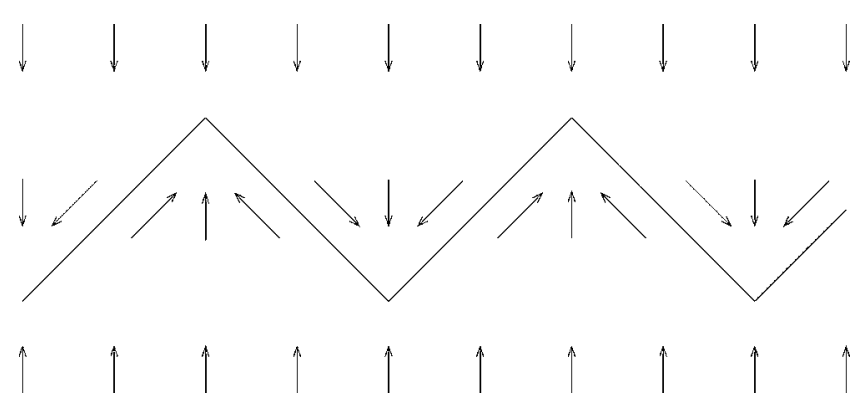

FIG. 1. The magnetic configuration in the Néel tail.

gation model, and thus ruling out explanations involving a cross-over with domain nucleation or other processes. ${ }^{2,9}$

The manuscript is organized as follows. In Sec. II we present an overview on the energetics of a zigzag domain wall, computing magnetostatic, anisotropy, and disorder energies. In Sec. III we estimate the mean zigzag half-period and the coercive field and compare our result with experimental observations, in order to test the reliability of our approximations. Next, in Sec. IV we present our model and the results obtained by Monte Carlo simulations for the frequency, temperature, and thickness dependence of the coercive field, and compare them with experiments. Our results are finally summarized in Sec. VI.

\section{ENERGETICS OF ZIGZAG DOMAIN WALLS}

In thin uniaxial ferromagnetic films, we can distinguish between two main classes of domain walls: the first is represented by prevalently straight (magnetically uncharged) walls parallel to the easy axis, and the second by charged walls separating two domains with head-on magnetization. Since there is a cost of magnetostatic energy associated with the magnetic charge that increases with the sample thickness, these walls are observed mostly in thin films. ${ }^{27}$ On the other hand, a charged straight wall is unstable and becomes metastable by forming a zigzag pattern to minimize its energy.

A quite extensive derivation of the equilibrium zigzag parameters (amplitude, period, and angle) has been reported in Ref. 32. The calculation is based on a Néel tail transition model $^{33}$ which involves an in-plane magnetization rotation over a transition region: the spins rotate following the wall shape. Thus the entire region surrounding the wall exhibits a nonuniform magnetization (see Fig. 1). This spreading occurs at the cost of increasing the anisotropy energy. The total energy may be expressed as a function of the zigzag parameters that are then obtained by minimization. From our point of view, it is important to stress that from magneto-optical images it can be inferred that the zigzag angle is constant across the wall and does not change during the motion. Thus, in the following, we will consider the angle as a fixed parameter in our model. As we mentioned in the Introduction, the zigzag shape of the wall is due to the interplay between the magnetostatic and the anisotropy contributions to the total energy. ${ }^{28}$ The magnetostatic term opposes a straight wall, which would maximize the magnetic charge density, and favors a large zigzag amplitude, so that the magnetic charges at the wall (all of the same sign) are as separated as possible.

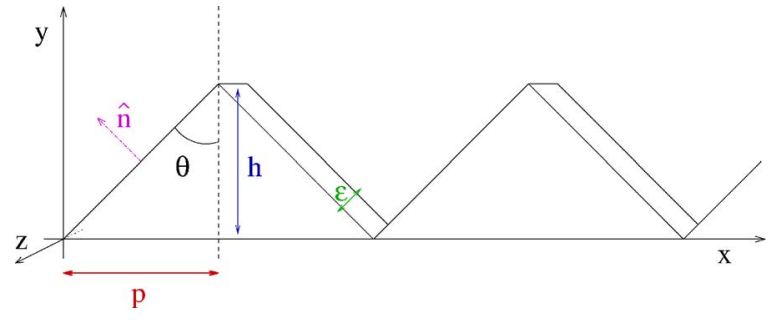

FIG. 2. (Color online) Sketch of the parameters of the zigzag wall. The easy axis is along the $y$ direction.

The anisotropy term prevents the amplitude to increase freely, avoiding an excessive deviation of the magnetization from the easy axis associated with a spread out Néel tail.

Our purpose is to study domain wall motion under an external magnetic field, by discrete model simulations. To this end, we calculate the total energy of an arbitrary zigzag wall configuration. As we are interested in the macroscopic response, we do not consider the details of the wall internal structure, and treat only the magnetostatic, the anisotropy and the disorder contributions

$$
E=E_{\mathrm{m}}+E_{\mathrm{an}}+E_{\mathrm{dis}} .
$$

In Eq. (1), the magnetostatic term $E_{\mathrm{m}}$ takes into account the interaction between magnetization and stray field, the anisotropy $E_{\text {an }}$ is the energy cost of deviations from easy axis, and $E_{\text {dis }}$ models structural disorder, impurities, defects, and so on. In the following subsections we will discuss these terms in more detail.

\section{A. Magnetostatic energy calculation}

We consider two generic segments of a zigzag wall of total length $L$. We label the segments as $i=1, \ldots, n$, where $n=L / p$ and $p$ is the half-period of the zigzag. We call $h$ the zigzag amplitude and $\theta$ the angle between the zigzag segment, and the easy axis. The thickness of the film is $\epsilon$, and it coincides with the wall thickness as we will consider only rigid walls (see Fig. 2 for a definition of the parameters).

Since the magnetostatic self-energy only depends on the total magnetic charge, which is constant during the wall motion, we limit the calculation to the magnetostatic interaction energy (i.e., $i \neq j$ ). The contribution due to the $i$ th and the $j$ th segments may be written as the surface integral

$$
E_{i j}=\int \sigma \phi_{i}(x, y, z) d S_{j},
$$

where $\sigma=\mathbf{M} \cdot \hat{\mathbf{n}}=2 M_{s} \sin \theta$ is the (constant) surface magnetic charge density ( $M_{s}$ is the saturation magnetization), $\hat{\mathbf{n}}$ is the unit vector normal to wall segment surface, and $S_{j}$ is the surface of the $j$ th segment. The scalar potential generated by the $i$ th segment, $\phi_{i}(x, y, z)$, is calculated by

$$
\phi_{i}(x, y, z)=\int \frac{\sigma}{\left|\mathbf{r}-\mathbf{r}^{\prime}\right|} d S_{i}^{\prime} .
$$

So, for $\epsilon$ small with respect to the segments distance, we can write 


$$
\begin{aligned}
E_{i j}= & 8 M_{s}^{2} \epsilon^{2} \mu_{0} \int_{j p}^{(j+1) p} d x \int_{i p}^{(i+1) p} d x^{\prime} \\
& \times \frac{1}{\sqrt{\left(x-x^{\prime}\right)^{2}+\left(m_{i} x+q_{i}-m_{j} x^{\prime}-q_{j}\right)^{2}}},
\end{aligned}
$$

where $m_{i}$ and $m_{j}$ are the slopes [which values should be $\pm h / p= \pm \tan (\pi / 2-\theta)]$ and $q_{i}$ and $q_{j}$ the $y$ intercepts of the $i$ th and $j$ th segments. The direct solution of Eq. (2) is very involved, and we report it in the Appendix.

\section{B. Anisotropy energy calculation}

The anisotropy energy term $E_{\text {an }}$ describes the energy cost of the deviation of magnetic moments from the easy axis of the material, which in the simple case of an uniaxial crystal can be written as

$$
E_{\text {an }}=\int d^{3} r K_{u} \sin ^{2} \phi
$$

where $K_{u}$ is the in-plane uniaxial anisotropy constant and $\phi$ is the angle between the easy axis and the magnetization vector. The rotation of the magnetization is associated with the Néel tail (Fig. 1). We assume ${ }^{28}$ that the charge is uniformly distributed over the entire band containing the wall. Although this approximation exaggerates the diffuseness of the charge, it has been used to calculate vertex angles and zigzag amplitudes that resulted to be in reasonable agreement with experimental observations. ${ }^{28}$ We can estimate Eq. (3) (with the notation sketched in Fig. 2) and obtain

$$
E_{\mathrm{an}}=\frac{\epsilon K_{u}}{h \tan \theta} \int_{-h / 2}^{h / 2} d y \int_{-(h / 2-y) \tan \theta}^{(h / 2-y) \tan \theta} d x \sin ^{2} \phi(x, y) .
$$

Assuming that $\phi(x, y)$ describes a linear in-plane rotation of the magnetization vector $\phi(x, y)=\theta x /[(h / 2-y) \tan \theta]$, developing $\sin ^{2} \phi$ in power series, and then integrating term by term, we obtain the anisotropy energy for unit length as

$$
E_{\text {an }}=\epsilon K_{u} h c(\theta),
$$

where $c(\theta)$ is a constant function of the zigzag angle $\theta$ :

$$
c(\theta)=\sum_{m, l=0}^{\infty} \frac{(-1)^{m, l}}{(2 m+1) !(2 l+1) !} \frac{\theta^{2(m+l+1)}}{2(m+l+1)+1},
$$

which could be evaluated numerically.

\section{Disorder}

Different sources of inhomogeneities are found in virtually all ferromagnetic materials, and the presence of structural disorder is essential to understand the hysteretic behavior, especially to account for the residual coercive field at $\omega \rightarrow 0$. Disorder is provided by vacancies, nonmagnetic impurities, dislocations, or grain boundaries in crystalline systems, variations of the easy axis between different grains for polycrystalline materials and internal stresses for amorphous alloys. We will consider only quenched (frozen) disorder, that does not evolve on the timescale of the magnetization reversal. For simplicity we model disorder by an energy contribution associated to each site which may be occupied by a segment (our discrete unit length) of the zigzag wall. This term is extracted from an uncorrelated random Gaussian distribution with zero mean.

The dependence of the disorder energy on the film thickness can be obtained by simple considerations. Given that the minimal segment has length $a$, we discretize the sample thickness in $\epsilon / a$ elements and associate a Gaussian random variable to each of them. The mean square value of the disorder energy per unit domain wall length is then proportional to the sum of contributions over the whole thickness, and thus to $\epsilon$. As a consequence of this, the disorder contributes to the total energy [Eq. (1)] by a term proportional to the square root of the film thickness

$$
E_{\mathrm{dis}}=\sqrt{\epsilon} \Delta
$$

where $\Delta$ is the variance of the random variables.

\section{THEORETICAL CONSIDERATIONS AND COMPARISON WITH MATERIALS}

\section{A. Zigzag parameters}

A way to test the reliability of our model is to compare the results of our model for some relevant parameters with the measured experimental values. As an example, we can estimate the typical zigzag half-period $p_{\text {eq }}$ for an equilibrium configuration in absence of external field. An approximation of the magnetostatic energy can be obtained in closed form by developing Eq. (2) for $p \ll L$ and is given, for unit length, by

$$
E_{\mathrm{m}} \simeq 8 \mu_{0} M_{s}^{2} \epsilon^{2} \ln (L / p) .
$$

In the absence of disorder and for $H_{\text {ext }}=0$, the total energy [Eq. (1)] can be written as

$$
E=E_{\mathrm{m}}+E_{\mathrm{an}} \simeq 8 M_{s}^{2} \epsilon^{2} \mu_{0} \ln (L / p)+\epsilon K_{u} \frac{p}{\tan \theta} c(\theta),
$$

where we have imposed $h=p / \tan \theta$. If the configuration is stable, considering the $T=0$ case, we can impose $\partial E / \partial p=0$ and obtain

$$
-\frac{8 M_{s}^{2} \epsilon^{2} \mu_{o}}{p}+\frac{\epsilon K_{u} c(\theta)}{\tan \theta}=0,
$$

from which follows

$$
p_{\text {eq }}=\frac{8 M_{s}^{2} \epsilon \mu_{0} \tan \theta}{K_{u} c(\theta)} .
$$

We can estimate the numerical value of $p_{\text {eq }}$ by using the parameters reported in literature for typical ferromagnetic thin films. For example, for Fe/GaAs(001) analyzed in Ref. 9 we can set $\mu_{0} M_{s}=2 T$ and $K_{u}=0.5 \times 10^{5} \mathrm{~J} / \mathrm{m}^{3}$, so that for a thickness $\epsilon=25 \mathrm{~nm}$ and an angle $\theta=20^{\circ}$, we obtain

$$
p_{\mathrm{eq}} \simeq 100 \mu \mathrm{m},
$$

which is in good agreement with the typical lengthscale inferred from magneto-optical investigations. ${ }^{9}$ 


\section{B. Coercive field and thickness dependence}

Another quantity that is interesting to obtain quantitatively is the value of the (zero frequency) coercive field. A very rough order of magnitude estimate could be obtained supposing that the disorder is small and that the anisotropy and magnetostatic terms are of the same order of magnitude. Close to the coercive field when the energy variation is stationary we can set

$$
\mu_{0} M_{s} \epsilon p h H_{c}=\epsilon p h K_{u} c(\theta),
$$

which implies

$$
H_{c}=\frac{K_{u} c(\theta)}{\mu_{0} M_{s}} .
$$

Employing the parameter values reported above, we obtain $H_{c} \sim 15 \mathrm{Oe}$, which is in reasonable agreement with the range of values found for $\mathrm{Fe} / \mathrm{GaAs}$ thin films. ${ }^{9}$

A more refined quantitative calculation of $H_{c}$ is difficult because we can not easily quantify the value of the disorder term. Nevertheless, we can obtain from simple considerations the thickness dependence of coercive field. The energy difference between two zigzag wall configurations in the presence of external magnetic field $H_{\text {ext }}$ is given by [see Eq. (1)]:

$$
\Delta E=\Delta E_{\mathrm{m}}+\Delta E_{\mathrm{an}}+\Delta E_{\mathrm{dis}}+\Delta E_{\mathrm{ext}},
$$

where the interaction $\Delta E_{\text {ext }}$ with $H_{\text {ext }}$ is given by

$$
\Delta E_{\mathrm{ext}}=-2 \mu_{0} M_{s} \epsilon H_{\mathrm{ext}} \Delta A,
$$

and $\Delta A$ is the area interested by the magnetization reversal. As it could be seen from Eqs. (2), (5), and (6), Eq. (9) could be rewritten as

$$
\Delta E=\epsilon^{2} \Delta E_{\mathrm{m}}^{\prime}+\epsilon \Delta E_{\mathrm{an}}^{\prime}+\sqrt{\epsilon} \Delta E_{\mathrm{dis}}^{\prime}+\epsilon \Delta E_{\mathrm{ext}}^{\prime},
$$

where $\Delta E_{\mathrm{m}}^{\prime}=\Delta E_{\mathrm{m}} / \epsilon^{2}, \Delta E_{\mathrm{an}}^{\prime}=\Delta E_{\mathrm{an}} / \epsilon, \Delta E_{\mathrm{dis}}^{\prime}=\Delta E_{\mathrm{dis}} / \sqrt{\epsilon}$, and $\Delta E_{\text {ext }}^{\prime}=\Delta E_{\text {ext }} / \epsilon$ do not dependent on $\epsilon$. As $\Delta E$ represents the energy barrier that the zigzag wall has to overcome in order to move in the direction of the external magnetic field, we expect that Eq. (11) encodes crucial informations on the dynamics.

The coercive field dependence on the thickness, can be obtained comparing $\Delta E_{\text {ext }}$ with the most relevant of the various terms contributing to $\Delta E$. For $\epsilon \rightarrow 0, \Delta E$ is dominated by the disorder contribution. Comparing $\Delta E_{\text {ext }} \propto \epsilon$ with $\Delta E_{\text {dis }}$ $\propto \sqrt{\epsilon}$, we easily obtain $H_{c} \propto 1 / \sqrt{\epsilon}$. If the wall is strongly pinned by disorder or anisotropy, starting from a saturated configuration, small external field changes are not able to trigger avalanches, resulting in square-shaped hysteresis loops with high coercitivity. Otherwise, if the pinning is weak, avalanches are induced even by relatively small field changes, so that the loops will be tighter, with small coercitivity. We can expect that the $1 / \sqrt{\epsilon}$ dependence will disappear in the limit of weak disorder regime. For intermediate $\epsilon$ values, the leading terms in Eq. (11) will be the anisotropy energy $\Delta E_{\mathrm{an}} \propto \epsilon$ and the interaction with the external magnetic field $\Delta E_{\text {ext }}$, leading to a thickness independent coercive field given by Eq. (8). Finally, for larger values of $\epsilon$, the dipolar energy will lead the energy barrier balance, inducing

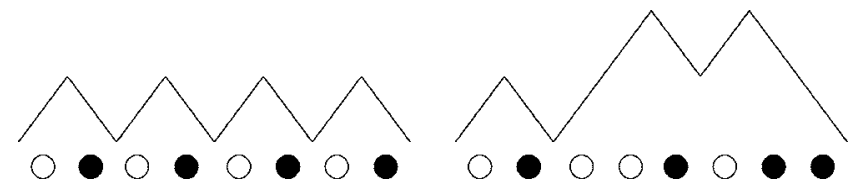

FIG. 3. The mapping. Two examples of zigzag configuration.

a linear dependence thickness dependence of the coercive field $\left(H_{c} \propto \epsilon\right)$. As it is shown in the following sections, these general considerations are confirmed by numerical simulations.

\section{MONTE CARLO SIMULATIONS}

\section{A. The model}

To investigate dynamic hysteresis, we perform Monte Carlo simulations based on the energy terms derived above. We consider a wall of length $L$ in a sample of finite dimensions. Since we are interested in macroscopic effects, we discretize the wall defining a minimal segment length $a$ and map the zigzag wall into a particle model. We define the minimal elements with negative slope as a particle and those with positive slope as a void, as sketched in Fig. 3. Thus we reduce the two-dimensional problem of a zigzag wall into a one-dimensional particle model evolving under the appropriate dynamic rules.

The Monte Carlo dynamics is implemented by choosing randomly an active pair of nearest neighbor elements, i.e., a particle-void or a void-particle pair, and trying to exchange their positions between each other. This rule corresponds to allow only the motion of segments with down-up or up-down slope, preserving the zigzag (solid-on-solid) structure of the wall. Once a possible displacement has been attempted, we calculate the energy difference [see Eq. (9)] between the starting configuration and the new one. If $\Delta E \leq 0$ we accept directly the move, otherwise the move is accepted with probability $P=\exp \left[-\Delta E / k_{B} T\right]$. In practice, the various contributions to $\Delta E$ are evaluated as follows.

$\Delta E_{\mathrm{m}}$ is obtained from Eq. (2). To simplify the expression, we can use $E_{i j}=1 / r_{i j}$ if the particles are not nearest neighbor, and the whole expression in Eq. (A1) otherwise. As it is discussed in the Appendix $E_{i j}$ deviates significantly from $1 / r_{i j}$ only if $i$ and $j$ are nearest neighbor. We can then perform another simplification, by absorbing the deviation from $1 / r_{i j}$ into the anisotropy (nearest-neighbor) term. In summary we can set $E_{i j}$ equal to $8 \mu_{0} M_{s}^{2} \epsilon^{2} / r_{i j} \forall i, j$, and renormalize the anisotropy term by an appropriate magnetostatic constant. Notice that $\Delta E_{m}$ is an attractive long range term, tending to aggregate all the particles. This configuration would correspond to a single period of a zigzag with $p$ $=L / 2$.

$\Delta E_{\text {an }}$ is a nearest-neighbor repulsive term that favors configurations where particles are followed by voids. This prevents the formation of a zigzag with a wide amplitude. In practice this term is treated as a positive contribution if the left (right) nearest neighbor of the segment $i(i+1)$ to be flipped has opposite slope with respect to $i(i+1)$, and a vanishing contribution otherwise. In this way we treat the 


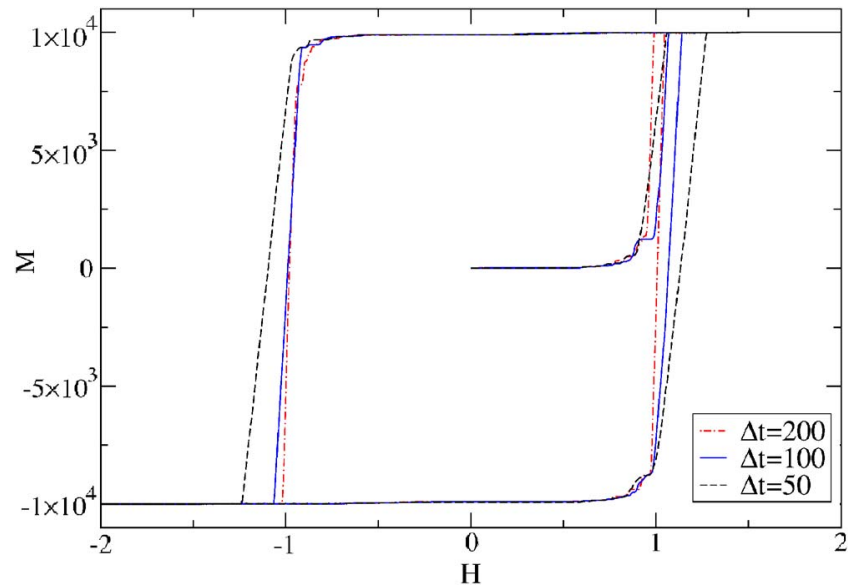

FIG. 4. (Color online) Some $T=0$ hysteresis cycles for different external field time rate.

rotation of the magnetic moments close to the zigzag wall, as uniformly distributed in a "band" surrounding the wall. ${ }^{28}$

$\Delta E_{\text {dis }}$ represents the contribution from structural disorder, whose time-independent intensity is randomly extracted with Gaussian distribution with zero mean for every site.

$\Delta E_{\text {ext }}$, the interaction with the external magnetic field $H_{\text {ext }}$, is calculated from Eq. (10), i.e., $\Delta E_{\text {ext }}=-2 \mu_{0} H_{\text {ext }} \Delta M$, where $\Delta M$ is the magnetization difference between the two configurations.

If the move is accepted we update the configuration and continue the process for a time interval $\Delta t$. In the spirit of the Monte Carlo method, each attempt corresponds to a time step $\tau=1 / N_{\text {act }}$, where $N_{\text {act }}$ is the number of active particles (i.e., those that are followed or preceded by a void). After each time interval $\Delta t$ is expired we increase the external field by $\Delta H_{\text {ext }}$ and restart the updating process. This rule corresponds to a field rate $\dot{H} \equiv \Delta H_{\text {ext }} / \Delta t \propto \omega$. We begin the simulation from the $M=0$ at $H_{\text {ext }}=0$ state and drive the sample to positive and then to negative saturation.

The results are presented in dimensionless units: distances are expressed in units of $L$, magnetization in units of $2 p h \epsilon M_{s}$, and fields in units $\mu_{0}$. Finally time is measured in Monte Carlo steps.

\section{B. Results}

\section{Rate dependence}

The first issue to be addressed in dynamic hysteresis is clearly the effect of the external field rate on the hysteresis. In Fig. 4, we show hysteresis loops obtained at $T=0$ for various rates. As expected from experiments and general considerations, small (high) rates correspond to narrow (large) cycles. To quantify this observation we can focus on the coercive field behavior. In Fig. 5 we show the dependence of $H_{c}$ on the field rate $d H / d t=\dot{H}$.

At $T=0$ (for a discussion of the behavior at non vanishing temperatures, see the following subsection), for the considered field time rates, $H_{c}$ shows an increasing linear dependence on $\dot{H}$, of the form $H_{c}=H_{p}+A \dot{H}$, where $A$ is a constant.
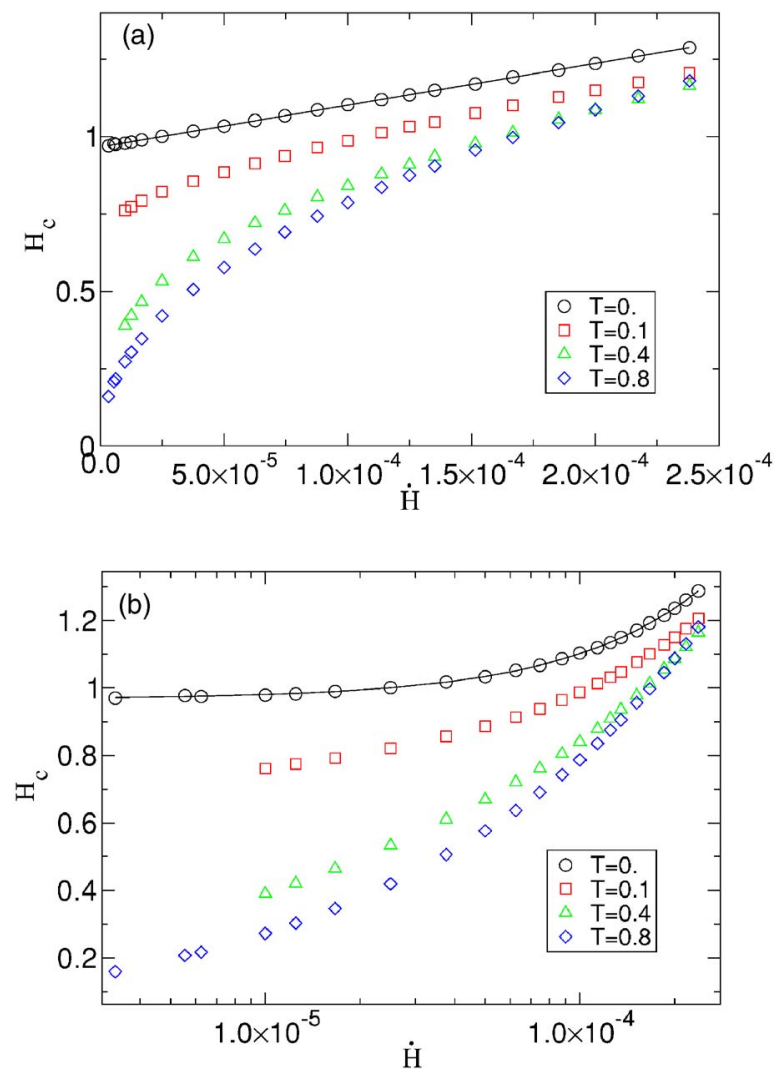

FIG. 5. (Color online) Coercive field vs external field rate at various temperatures, in a linear (a) and semilog plot (b). Every value is mediated over 1000 realizations corresponding to different disorder configurations. The solid lines on the $T=0$ curves are a linear fit.

This means that in the adiabatic limit (low rates) $H_{c}$ goes to a nonvanishing value $H_{p}$ [as clearly shown in the semilogarithmic plot of Fig. 5(b)], that we can interpret as the pinning dominated quasistatic component due to structural disorder and anisotropy, while the linear behavior of $H_{c}$ in the high rates regime represents the domain wall dominated dynamic contribution.

This result is a particular case $(\alpha=1)$ of the law

$$
H_{c}=H_{p}+A \dot{H}^{\alpha},
$$

used to fit simulations and experiments in Refs. 18, 19, and 26 (but with a different exponent $\alpha$ ) and suggested by the theory presented in Ref. 20. In the model of Ref. 20, the exponent $\alpha$ is related to the scaling exponent $\beta$ associated with the depinning transition of the domain wall. In particular, it is assumed that under a constant applied field the domain wall velocity $v \propto d M / d t$ follows:

$$
v=C\left(H-H_{p}\right)^{\beta},
$$

for $H$ slightly larger than $H_{p}$, while $v$ vanishes for $H<H_{p}$. Using Eq. (13) as a constitutive law, one can readily show that the dynamic coercive field scales as in Eq. (12) with $\alpha$ $=1 /(\beta+1)$. The limit $\alpha=1$ corresponds to $\beta \rightarrow 0$ or else to a gap in the domain wall velocity as a function of the external magnetic field. A very sharp dependence of the velocity on 


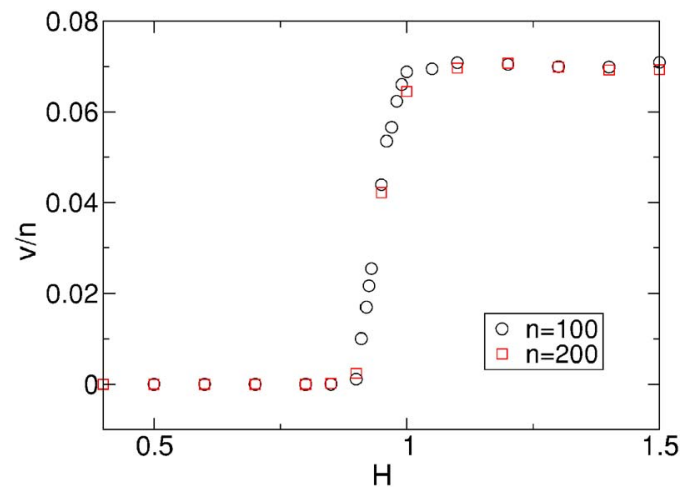

FIG. 6. (Color online) Velocity of the domain wall, normalized to the number of unit segments of the zigzag, as a function of an external constant field. Each point is an average over 2000 different disorder configurations.

the field is indeed observed in our model (see Fig. 6) around the coercive field, where the segments of the zigzag wall begin to move. This is probably a strong pinning effect: due to the zigzag structure of the wall the system is trapped by strong anisotropy barriers and collective effects, typically leading to a continuous depinning, are suppressed.

\section{Temperature dependence}

Another interesting issue to analyze is the effect of temperature $T$ on dynamic hysteresis. Even if thermal effects do not seem to be relevant for most ferromagnetic thin films where $k_{B} T \ll \Delta E$, as it can be checked, e.g., comparing $k_{B} T$ $\simeq 10^{-21} \mathrm{~J}$ at room temperature, where the experiments are typically performed, with an estimate of $\Delta E \simeq E_{\text {an }} \simeq 10^{-12} \mathrm{~J}$ which is obtained with the parameters of $\mathrm{Fe} / \mathrm{GaAs}(001)$ given in Sec. III. Thermal activation could, however, play a role at very low frequencies or in ultrathin ferromagnetic films. In general terms, the increase of the temperature (at reasonably low rate, see Fig. 8) acts on the hysteresis cycles shape in an similar way as the decrease of the rate (see Fig. 7). Since a temperature increase enhances the probability for the wall to overcome energy barriers, at high (low) tempera-

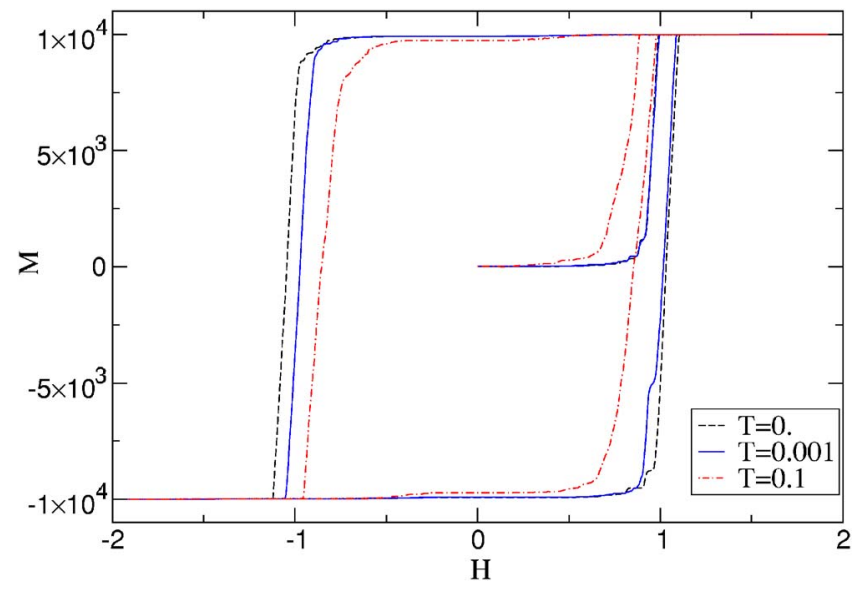

FIG. 7. (Color online) Some hysteresis cycles for various temperature. The external fixed field time rate is $\Delta t=100$.

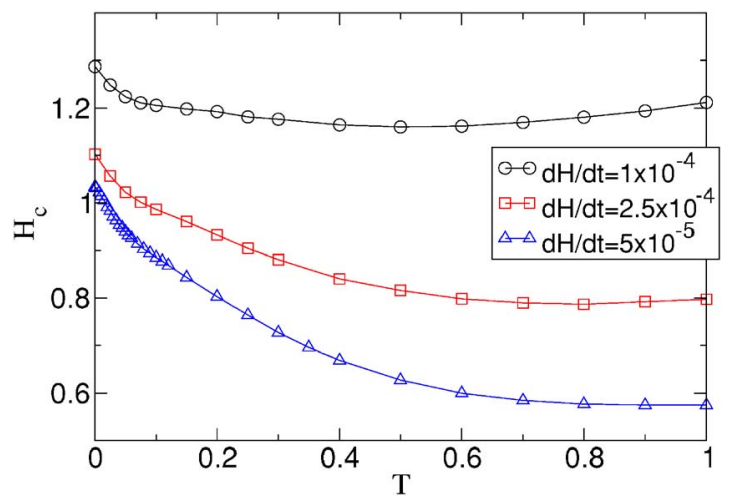

FIG. 8. (Color online) Coercive field vs temperature for various external field time rates. Every value is mediated over 1000 realizations corresponding to different disorder configurations.

ture hysteresis loops will be large (narrow). However at very high frequencies, the system is not able to readily respond to the external field, and the decreasing dependence of $H_{c}$ on increasing temperatures is violated, as shown in Fig. 8. This explains the crossover between the curves with $T=0.4$ and $T=0.8$ (Fig. 5).

In Fig. 5 we show the dependence of $H_{c}$ vs $\dot{H}$ at various nonzero temperatures. It is interesting to note that our simulations predict that the high $T$ behavior of $H_{c}$ vs $\dot{H}$ deviates from the linear behavior established at $T=0$. This can be understood from general considerations, since when $T>0$ thermal activation will lead creep domain wall motion even for $H<H_{p}$. Hence, according to the theoretical analysis presented in Ref. 21 at low frequencies the dynamic coercive field will deviate from Eq. (12) and decay as $H_{c}$ $\sim 1 /[\ln (\dot{H})]^{1 / \mu}$ where $\mu$ is a creep exponent. This result is consistent with Fig. 5(b), although the limited scaling range does not allow for a quantitative confirmation.

It is worth noting that due to numerical simulations we cannot push the simulations to very low rates to test whether similar deviations are observed at $T=0$. On the other hand, we do observe deviations from the linear behavior as soon as thermal activation is introduced in the model.

\section{Thickness dependence}

Finally, we address the role of the film thickness in dynamic hysteresis. As it can be seen in Eq. (11), for sufficiently large $\epsilon$ above the purely disorder dominated regime discussed in Sec. III B, the energy barrier increases linearly with $\epsilon$ at $T=0$, and the coercive field does so as well.

Turning to a more quantitatively discussion, at $T=0$ the results of Eq. (11) are confirmed by the simulations for various $\epsilon$ summarized in Fig. 9. In the two upper curves, where the disorder energy term is not negligible, we notice three regimes: a divergence proportional to $1 / \sqrt{\epsilon}$ at very low $\epsilon$, due to the disorder term and linear regime for high $\epsilon$ values, due to the dipolar term (the regime independent from $\epsilon$ that would be due to the anisotropy energy and the external field, cannot be seen clearly in that figure). Moreover, from the lower curve of Fig. 9 (the one without disorder) we confirm that the low- $\epsilon$ divergence is due to the disorder term. Other- 


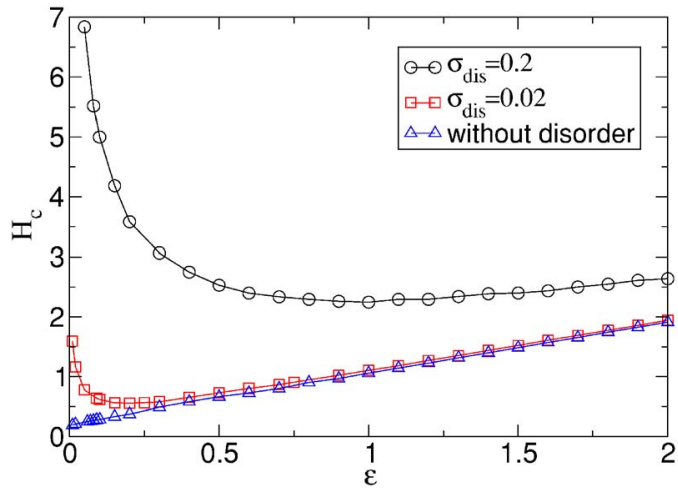

FIG. 9. (Color online) Coercive field vs film thickness for various values of the $\sigma$ of the disorder Gaussian distribution. Every value is mediated over 200 realizations corresponding to different disorder configurations.

wise, the thickness dependence of the coercive field does not affect the linearity of the rate dependence of $H_{c}$, which remains valid for every $\epsilon$ value, as is seen in Fig. 10 (at $T=0$ ).

\section{COMPARISON WITH EXPERIMENTS}

It is interesting to compare the predictions of our models with experiments. An extensive recording of dynamic hysteresis in a material with a single zigzag domain wall was reported in Ref. 9 for two different thickness values. Here, for the sake of comparison, we report this data in Fig. 11 both in linear and in semilogarithmic scales. The logarithmic scale can be misleading because an additive constant, such as $H_{p}$ in Eq. (12) leads to a flattening of the curve and may suggest that a crossover is taking place. This has been interpreted in Ref. 9 as due to the interplay between nucleation and domain wall propagation. We have tried to fit the experimental data with Eq. (12) but the result is not entirely satisfactory. A direct fit over the entire range yields $\alpha=1$ for the $25 \mathrm{~nm}$ sample and $\alpha=0.76$ for the $5 \mathrm{~nm}$ one. However, if we restrict the fit to the low rates region, the exponent appears to be

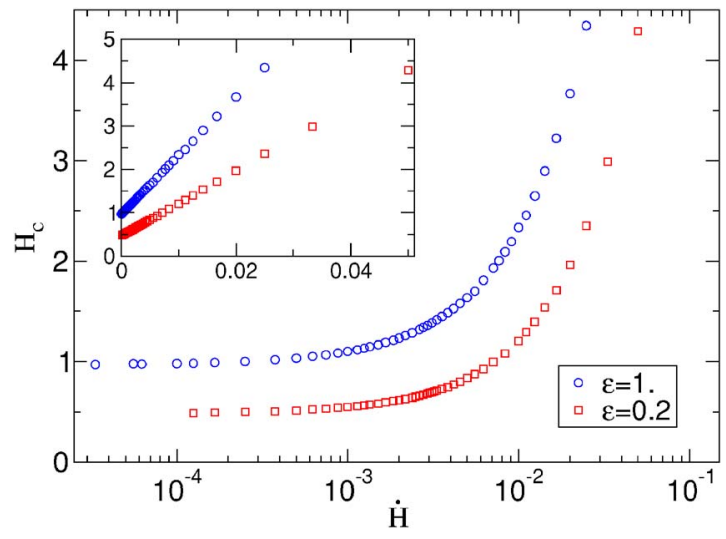

FIG. 10. (Color online) Coercive field vs rate $\dot{H}$ at $T=0$ for two different thicknesses in semilogarithmic scale. In the inset: a linear plot of the same data. The linear behavior is not affected by the thickness. Compare the present figure with the experimental results reported in Fig. 11.

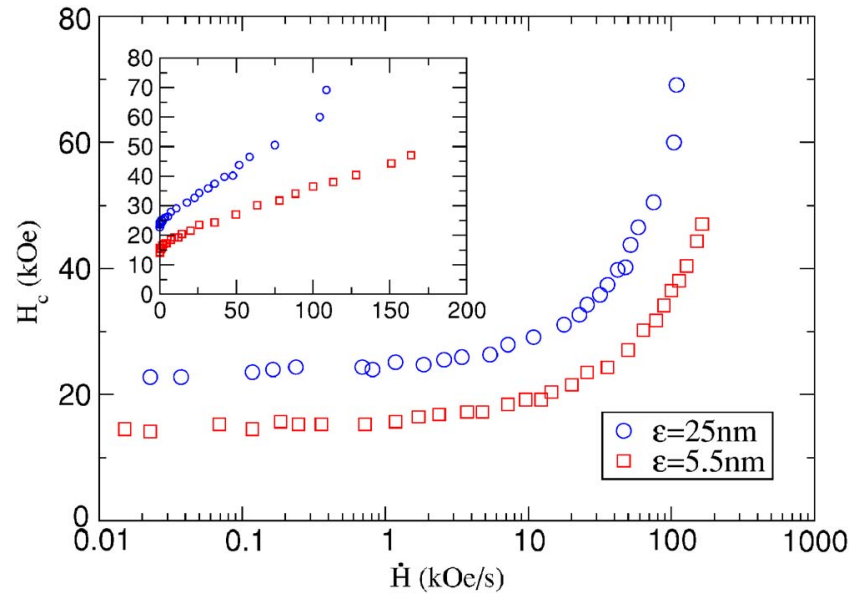

FIG. 11. (Color online) Coercive field as a function of the external field rate from experiments on $\mathrm{Fe} / \mathrm{GaAs}$ thin films for two different thicknesses in semilogarithmic scale. In the inset: a linear plot of the same data. The deviation from linear behavior could be due to thermal activation as in our model. Data are obtained from Ref. 9.

smaller, and closer to $\alpha=0.5$. In summary, a linear regime could only be identified in the high rate region, with nonlinearities that are more marked for small rates and thicknesses.

If we compare the experimental results with our model, we notice that the value of $H_{c}$ for the thinner sample $(\epsilon$ $=55 \AA)$ is smaller than the value measured for the thicker one $(\epsilon=250 \AA)$ at the same field rate $\dot{H}$, and this behavior is correctly captured by our model (see Fig. 10). As for the rate dependence, we can not reach a satisfactory quantitative agreement, but we propose to interpret the nonlinearities at low rates by means of thermal activation (compare with Fig. 5). This interpretation is supported by the fact that nonlinearities are stronger for the thinner sample, as it would be expected if thermal effects where the cause. In Fig. 10 we show simulated results at $T=0$. In fact, as previously discussed (see Sec. IV B 2), temperature effects are expected to be small in ferromagnets, and thus the coercive field, for sufficiently high rates, for small (but nonzero) $T$ should be similar to the one obtained at $T=0$.

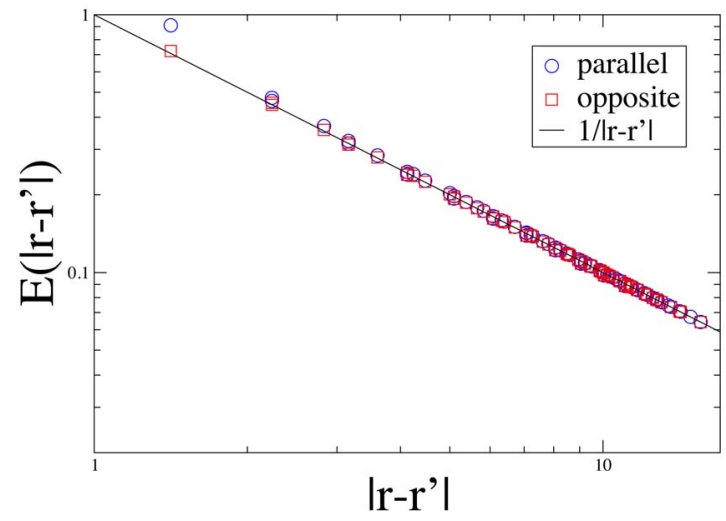

FIG. 12. (Color online) Interaction magnetostatic energy between two generic segments with parallel or opposite slopes as a function of the distance between their centers of mass. 
We notice as well that the data reported in Ref. 13 for even thinner films of $\mathrm{Fe} / \mathrm{GaAs}$ show a logarithmic decrease of $H_{c}$ at low rate, not observed for thicker samples where $H_{c}$ tends instead to a constant as in Fig. 11.

\section{CONCLUSIONS}

The dynamics of two dimensional ferromagnetic systems is still under debate, both on the theoretical and the experimental side. A crucial issue is the description of the dynamic hysteresis, that is related to power losses and thus plays an important role in several technological applications. Here, we have analyzed ferromagnetic thin films with zigzag domain walls, arising when the magnetization vectors in two nearest-neighbor domains meet head-on at the wall that separates them. To investigate dynamic hysteresis, we have studied the motion of zigzag domain walls by developing a simple discrete model based on the interplay between dipolar and anisotropy energy contributions, in presence of structural disorder. Under some simple approximations one can estimate some experimentally relevant quantities, such as the typical zigzag half-period and the coercive field, which turn out to be in quantitative agreement with experimental observations.

Although quite simplified, our model allows to recover the behavior of coercive field $H_{c}$ in dynamic hysteresis. We have studied the dependence of $H_{c}$ on the applied magnetic field rate $\dot{H}$ at $T=0$ and found that the coercive field scales as $H_{c}=H_{p}+A \dot{H}$. This linear behavior is in qualitative agreement with experiments, ${ }^{9}$ which we can thus explain by means of pure domain wall propagation model, without the need to invoke other dynamic processes as domain nucleation. We have also simulated hysteresis at $T>0$ and show that high temperature at low rate induces narrow loops and the coercive field decreases with respect to the $T=0$ case, while at high rate the situation is less intuitive due to the delay between the system response and the external driving field. We have also studied the dependence of the coercitivity from the film thickness $\epsilon$. The behavior indicated by the simulations is explained by simple analytical considerations. For small disorder, we find that the thickness does not affect the rate dependence of $H_{c}$ at $T=0$. It is interesting to remark that our model could be applicable to ferroelectric materials which are known to show as well zigzag domain walls. It would be very interesting to compare our results with experiments in this case as well.

\section{ACKNOWLEDGMENTS}

We would like to thank G. Durin and A. Magni for useful discussions.

\section{APPENDIX}

Analytical calculation of magnetostatic energy: the result of Eq. (2) is given by

$$
\begin{aligned}
E_{i j}= & 8 M_{s}^{2} \epsilon^{2}\left\{\delta ( m _ { i } , m _ { j } ) \left[g_{1}\left(m_{i}, q_{i}-q_{j}, m_{j}, j p, i p\right)\right.\right. \\
& -g_{1}\left(m_{i}, q_{i}-q_{j}, m_{j}, j p,(i+1) p\right)-g_{1}\left(m_{i}, q_{i}-q_{j}, m_{j},(j\right. \\
& \left.+1) p, i p)+g_{1}\left(m_{i}, q_{i}-q_{j}, m_{j},(j+1) p,(i+1) p\right)\right]+\delta\left(m_{i},\right. \\
& \left.-m_{j}\right)\left[g_{2}\left(m_{i}, q_{i}-q_{j}, m_{j}, j p, i p\right)-g_{2}\left(m_{i}, q_{i}-q_{j}, m_{j}, j p,(i\right.\right. \\
& +1) p)-g_{2}\left(m_{i}, q_{i}-q_{j}, m_{j},(j+1) p, i p\right)+g_{2}\left(m_{i}, q_{i}\right. \\
& \left.\left.\left.-q_{j}, m_{j},(j+1) p,(i+1) p\right)\right]\right\},
\end{aligned}
$$

where

$$
\begin{gathered}
g_{1}\left(m, q-q^{\prime}, m, x, x^{\prime}\right)=\frac{1}{1+m^{2}}\left\{\left|\mathbf{r}-\mathbf{r}^{\prime}\right|-\frac{a\left(\mathbf{r}-\mathbf{r}^{\prime}\right)}{2} \ln \left[\frac{\left|\mathbf{r}-\mathbf{r}^{\prime}\right|-a\left(\mathbf{r}-\mathbf{r}^{\prime}\right)}{\left|\mathbf{r}-\mathbf{r}^{\prime}\right|+a\left(\mathbf{r}-\mathbf{r}^{\prime}\right)}\right]\right\} \quad \forall q \neq q^{\prime}, \\
g_{1}\left(m, q-q^{\prime}, m, x, x^{\prime}\right)=\frac{\left(-x+\left(x-x^{\prime}\right) \ln \left(x-x^{\prime}\right)\right)}{\sqrt{1+m^{2}}} \quad \text { if } q=q^{\prime} \\
g_{2}\left(m, q-q^{\prime}, m, x, x^{\prime}\right)=b\left(\mathbf{r}-\mathbf{r}^{\prime}\right) \ln \left[\frac{\left|\mathbf{r}-\mathbf{r}^{\prime}\right|+a\left(\mathbf{r}-\mathbf{r}^{\prime}\right)}{\left|\mathbf{r}-\mathbf{r}^{\prime}\right|-a\left(\mathbf{r}-\mathbf{r}^{\prime}\right)}\right]+b\left(\mathbf{r}^{\prime}-\mathbf{r}\right) \ln \left[\frac{\left|\mathbf{r}-\mathbf{r}^{\prime}\right|+c\left(\mathbf{r}-\mathbf{r}^{\prime}\right)}{\left|\mathbf{r}-\mathbf{r}^{\prime}\right|-c\left(\mathbf{r}-\mathbf{r}^{\prime}\right)}\right]
\end{gathered}
$$

and

$$
\begin{gathered}
a\left(\mathbf{r}-\mathbf{r}^{\prime}\right)=\frac{x^{\prime}-x+m^{2}\left(x^{\prime}-\frac{m^{\prime}}{m} x\right)-m^{\prime}\left(q-q^{\prime}\right)}{\sqrt{1+m^{2}}}, \\
b\left(\mathbf{r}-\mathbf{r}^{\prime}\right)=\frac{q-q^{\prime}+2 m x}{4 m \sqrt{1+m^{2}}}, \\
c\left(\mathbf{r}-\mathbf{r}^{\prime}\right)=\frac{x-x^{\prime}+m^{2}\left(x+x^{\prime}\right)+m\left(q-q^{\prime}\right)}{1+m^{2}},
\end{gathered}
$$


and $\delta\left(m, m^{\prime}\right)$ is Kronecker's delta.

In Fig. 12 we plot the function $E_{i j}$ (for unitary $8 M_{s}^{2} \epsilon^{2}$ ) as a function of the distance between the centers of mass of the segments $i$ and $j$. As could be seen, the value of magneto- static interaction energy is mainly proportional to $1 /\left|\mathbf{r}-\mathbf{r}^{\prime}\right|$ $=1 / r_{i j}$ for each pair of $i$ and $j$ but the nearest neighbor, where magnetic charges are sufficiently close to each other to experience the very shape of their spatial distribution.
${ }^{1}$ B. K. Chakrabarti and M. Acharyya, Rev. Mod. Phys. 71, 847 (1999).

${ }^{2}$ T. A. Moore and J. A. C. Bland, J. Phys.: Condens. Matter 16, R1369 (2004).

${ }^{3}$ G. Bertotti, Hysteresis in Magnetism (Academic Press, San Diego, 1998).

${ }^{4}$ Y.-L. He and G.-C. Wang, Phys. Rev. Lett. 70, 2336 (1993).

${ }^{5}$ C. N. Luse and A. Zangwill, Phys. Rev. E 50, 224 (1994).

${ }^{6}$ Q. Jiang, H.-N. Yang, and G.-C. Wang, Phys. Rev. B 52, 14911 (1995).

${ }^{7}$ B. Raquet, R. Mamy, and J. C. Ousset, Phys. Rev. B 54, 4128 (1996).

${ }^{8}$ J.-S. Suen and J. L. Erskine, Phys. Rev. Lett. 78, 3567 (1997).

${ }^{9}$ W. Y. Lee, B.-Ch. Choi, Y. B. Xu, and J. A. C. Bland, Phys. Rev. B 60, 10216 (1999).

${ }^{10}$ B. C. Choi, W. Y. Lee, A. Samad, and J. A. C. Bland, Phys. Rev. B 60, 11906 (1999).

${ }^{11}$ W. Y. Lee, Y. B. Xu, S. M. Gardiner, and J. A. C. Bland, J. Appl. Phys. 87, 5926 (2000).

${ }^{12}$ W. Y. Lee, A. Samad, T. A. Moore, J. A. C. Bland, and B. C. Choi, Phys. Rev. B 61, 6811 (2000).

${ }^{13}$ T. A. Moore, J. Rothman, Y. B. Xu, and J. A. C. Bland, J. Appl. Phys. 89, 7018 (2001).

${ }^{14}$ X. Chen, O. Sichelschmidt, W. Kleemann, O. Petracic, C. Binek, J. B. Sousa, S. Cardoso, and P. P. Freitas, Phys. Rev. Lett. 89, 137203 (2002).

${ }^{15}$ G. Asti, M. Ghidini, and M. Solzi, J. Magn. Magn. Mater. 242245, 973 (2002).

${ }^{16}$ L. Santi, R. Sommer, A. Magni, G. Durin, F. Colaiori, and S. Zapperi, IEEE Trans. Magn. 39, 2666 (2003).
${ }^{17}$ J.-S. Suen, M. H. Lee, G. Teeter, and J. L. Erskine, Phys. Rev. B 59, 4249 (1999).

${ }^{18}$ Fan Zhong, Jin Xin Zhang, and Xiao Liu, Phys. Rev. E 52, 1399 (1995).

${ }^{19}$ F. Zhong, J. Dong, and D. Y. Xing, Phys. Rev. Lett. 80, 1118 (1998).

${ }^{20}$ I. F. Lyuksyutov, T. Nattermann, and V. Pokrovsky, Phys. Rev. B 59, 4260 (1999).

${ }^{21}$ T. Nattermann, V. Pokrovsky, and V. M. Vinokur, Phys. Rev. Lett. 87, 197005 (2001).

${ }^{22}$ J. P. Sethna, K. Dahmen, S. Kartha, J. A. Krumhansl, B. W. Roberts, and J. D. Shore, Phys. Rev. Lett. 70, 3347 (1993).

${ }^{23}$ H. L. Richards, M. A. Novotny, and P. A. Rikvold, Phys. Rev. B 54, 4113 (1996).

${ }^{24}$ S. W. Sides, P. A. Rikvold, and M. A. Novotny, Phys. Rev. E 57, 6512 (1998).

${ }^{25}$ S. Zapperi, P. Cizeau, G. Durin, and H. E. Stanley, Phys. Rev. B 58, 6353 (1998)

${ }^{26}$ C. Nistor, E. Faraggi, and J. L. Erskine, Phys. Rev. B 72, 014404 (2005).

${ }^{27}$ A. Hubert and R. Schäfer, Magnetic domains (Springer, New York, 1998).

${ }^{28}$ M. J. Freiser, IBM J. Res. Dev. 23, 330 (1979).

${ }^{29}$ N. Curland and D. E. Speliotis, J. Appl. Phys. 41, 1099 (1970).

${ }^{30}$ D. Dressler and J. Judy, IEEE Trans. Magn. 10, 674 (1974).

${ }^{31}$ A. N. Alexeyev, Appl. Phys. Lett. 68, 159 (1996).

${ }^{32}$ H. L. Huang and T. H. Chen, J. Magn. Magn. Mater. 135, 89 (1994).

${ }^{33}$ L. Sanders, R. Jones, and A. Collins, J. Phys. D 10, 2503 (1997). 